\title{
E. W. Beth as a philosopher of physics
}

\author{
Dennis Dieks
}

Received: 7 April 2009 / Accepted: 12 September 2009 / Published online: 4 September 2010

(C) The Author(s) 2010. This article is published with open access at Springerlink.com

\begin{abstract}
This paper examines E. W. Beth's work in the philosophy of physics, both from a historical and a systematic point of view. Beth saw the philosophy of physics first of all as an opportunity to illustrate and promulgate a new and modern general approach to the philosophy of nature and to philosophy tout court: an approach characterized negatively by its rejection of all traditional metaphysics and positively by its firm orientation towards science. Beth was successful in defending this new ideology, and became its leading Dutch representative in the first two decades after the second world war. Beth also contributed importantly to the method of the philosophy of physics in a narrower sense, by proposing and promoting the semantic approach in the formal analysis of physical theories. Finally, he worked on several specific foundational questions; but he was probably too much of a logician to leave his mark in this area.
\end{abstract}

Keywords Beth · Philosophy of nature $\cdot$ Philosophy of physics · Logic · Quantum mechanics · Relativity

\section{Introduction}

In 1946, at the relatively young age of 37, Evert Willem Beth was appointed professor of Logic and its History, plus the Philosophy of the Exact Sciences at the University of Amsterdam. Beth's candidature had been staunchly supported ${ }^{1}$ by the influential Amsterdam physics professor Jacob Clay (1882-1955). Clay had been active as a

\footnotetext{
1 See for the controversies surrounding the Amsterdam professorship Van Berkel (1996, Chap. 8).

D. Dieks $(\bowtie)$

History and Foundations of Science, Utrecht University, P.O. Box 80.010, 3508 TA,

Utrecht, The Netherlands

e-mail: d.dieks@uu.nl
} 
philosopher himself, starting out as a Hegelian, but soon turning into the direction of logical empiricism: he had become involved in the Significs Circle surrounding Mannoury and was one of the first editors of the journal Synthese. Clay belonged to a group of philosophically interested physicists, like H. A. Kramers, A. D. Fokker and $\mathrm{Ph}$. Kohnstamm, who in the years between the two world wars repeatedly stressed the philosophical importance of recent developments in physics and sought public attention for this. Although modern philosophical investigations of mathematics had found acceptance in Dutch academia through the work of Brouwer, Mannoury and Heyting, academic philosophy had not seen a similar movement with regard to the study of the empirical sciences - in spite of the fact that, according to Clay, academic lectures in the logic of science had become an 'urgent requirement of our times' (Van Berkel 1996, p. 286). Evert Beth seemed the ideal candidate to fill this lacuna.

Evert Beth had been born in 1908 into a family in which both scientific and humanistic interests were prevalent. His father, H. J. E. Beth, was a mathematician who wrote widely on educational, historical and philosophical aspects of his discipline. In 1932, for example, Beth senior published a two-volume work on Newton's Principia in which he gave Dutch translations (together with the original Latin) of what he considered the most important passages in Newton's treatise and in which he provided these fragments with historical and philosophical annotations. An interesting example of the method he followed is in the chapter in which he deals with Newton's Scholium on space and time (Beth 1932, Chap. III). After having presented Newton's text, H. J. E. Beth proceeds to a discussion of relational alternatives to Newton's theory of absolute space, going from Huygens to Mach. He finally mentions that the whole issue has been resolved only recently by Einstein who has turned Mach's desiderata into a concrete physical theory- "nothing enables us better to understand both the depths and the limitations of Newton's conceptions than considering them in the light struck by our brilliant contemporary" (Beth 1932, p. 58). No doubt young Evert was thoroughly familiar with his father's ideas and underwent the influence of his outlook. ${ }^{2}$

After having finished secondary school Evert Beth studied mathematics and physics in Utrecht, where he graduated with distinction in 1932. In the meantime he had also been studying philosophy, and already in 1935 he defended a dissertation on the philosophy of mathematics (entitled Reason and Intuition in Mathematics (Beth 1935)). This work brought him into contact with G. Mannoury and other members of the Significs Circle that we already mentioned in connection with Jacob Clay. Beth's dissertation combined elements from neo-kantian (Marburg) philosophy with strands from logical empiricism (especially Carnap's work); Beth's association with kantianism was to fade away soon, however. In his 1938 article 'The conception of science of Nietzsche and that of the neo-positivists' (Beth 1938) he endorses the refutation of the synthetic a priori by the philosophers of the Vienna Circle, and points out that similar themes can already be found in Nietzsche's work; he even goes as far as declaring that all differences between Nietzsche's philosophy and neo-positivism pale before their all-important agreement about the need to reject metaphysics. He adds

\footnotetext{
2 This is confirmed by the fact that Evert Beth added a last-minute note to the original manuscript of Beth (1948a), drawing attention to a book by his father, on the history of gravitational theories, that had just appeared.
} 
that this anti-metaphysical philosophical outlook may be just what is needed by the 'modern scientific person' to find his way 'in the present confused political and social circumstances.'

In June 1944 Evert Beth finished the manuscript of Natuurphilosophie (Philosophy of Nature), whose publication in slightly adapted form followed in 1948, after the second world war (Beth 1948a). In this book, and to a lesser extent in remarks made in his more mathematics-oriented Philosophy of Space (Wijsgerige Ruimteleer) from 1950 (Beth 1950), we find Beth's mature views on the status of the philosophy of science, in particular the philosophy of physics; plus a number of concrete case studies in the foundations of physics. Beth's solid background in physics and mathematics, his significs sympathies and contacts, but certainly also his ideas about philosophy, science and their interrelation as now fully worked out in Natuurphilosophie, must have played a major role in his securing the Amsterdam professorship. It is on the contents of Natuurphilosophie that we shall concentrate in the following.

\section{Natuurphilosophie}

In the preface of Natuurphilosophie Beth writes: "One should not expect from this booklet an objective account of the present state of research in the philosophy of nature. I have always, whenever there was an opportunity, first of all mentioned and explained views that differ from mine, in order to make my own opinion stand out as clearly as possible...." (Beth 1948a, p. 7). This strategy goes some way towards explaining why of all contemporary philosophers of science the one mentioned and discussed most in Natuurphilosophie is P. Hoenen S.J. (1880-1961), a Dutch Jesuit priest who was professor of cosmology at the Gregorian university in Rome. Indeed, the register of names mentions Carnap, Clay, van Dantzig, Destouches-Février, Franck, Gentzen, Lukasiewicz, Meyerson and Reichenbach each only once, whereas Hoenen receives ten citations, of which two refer to discussions filling three consecutive pages. This asymmetry illustrates the pamphlet-like flavor of the work: Natuurphilosophie is meant to define and defend a new stance that radically breaks with traditional ideas, in this case the neothomist views of Hoenen.

Hoenen's philosophical ideas were indeed ideally suited to make stand out Beth's own position. Petrus Hoenen was a typical exponent of the campaign of catholic emancipation that took place in the Netherlands in the first half of the twentieth century. Although forming nearly one half of the total population, catholics had remained behind in social and cultural respects - a situation whose origins can be traced back to the effects of the Dutch Revolt in the sixteenth and seventeenth centuries. As one symptom of the general arrears it was noted by catholic leaders at the beginning of the twentieth century that catholic participation in scientific research was far below what could be expected on the basis of population numbers, and several initiatives were launched to redress this - with as one of the eventual results the establishment of the Catholic University of Nijmegen in 1923. It was in this climate of catholic emancipatory ideals that Petrus Hoenen S.J., after having finished his theological studies, started studying physics at the university of Leiden. In 1912 he defended an excellent dissertation on a thermodynamical subject there, supervised by H. A. Lorentz. After a teaching career at several Dutch seminaries he was finally appointed a professor of 
cosmology and philosophy of nature at the Gregorian university in Rome in 1924. Although now relatively far away from home, Hoenen remained interested and active in the Dutch catholic cause and tried to shore up the process of emancipation in his own peculiar way.

While studying the developments in modern physics, especially those in quantum theory, Hoenen had come to the conclusion that the new theories were in flagrant conflict with any mechanistic philosophy of nature and that there was only one reasonable alternative, flexible enough to accommodate the revolutionary changes: neothomism. As he concluded in his paper The great crisis of the physical sciences (Hoenen 1928), "Physical science will be Aristotelian-Thomist, or it will not be at all." Hoenen elaborated this viewpoint in a bulky Latin treatise, Cosmologia, published in Rome in 1930 (Hoenen 1930), which was first of all meant as a textbook for courses at the Gregoriana. He also wrote a companion volume in Dutch, Philosophy of Inorganic Nature (Hoenen 1940), first published in 1940. Both books were reprinted several times, and formed the basis of Beth's discussion of Hoenen in Natuurphilosophie. Hoenen further elaborated on the material covered in these books in several papers and frequently explained his position in popular lectures in Holland. The tenor of all these contributions was that there existed a typically catholic, thomistic philosophy of nature that was unique in its power to give an account of modern quantum theory and other recent scientific developments.

Beth confronts Hoenen in Chapters I and III of Natuurphilosophie, in which he discusses the general task and status of philosophy of nature. In line with the method used by his father in Beth (1932), a method he himself explains and recommends in his 1946 paper Historical studies in traditional philosophy (Beth 1646c), Beth starts by reviewing from a modern, analytical point of view the various views about the role of philosophy of nature that can be discerned in history. He argues that most traditional philosophical systems aspire either to criticize science, or to give a foundation to science, or to complete science, for example with 'deeper explanations'. In this sense, these philosophies of science position themselves on the same level of discourse as science itself. Hoenen's philosophy of science is an example par excellence: Hoenen regards modern physical theories as a 'specification' of the general principles laid down by Aristotle and Aquinas. Clearly, this is a relation between science and philosophy that deprives science from at least some of its freedom: it will not be possible for science to take any form whatever and still remain within thomistic confines. Indeed, as Beth points out, Hoenen criticized the special theory of relativity for abandoning absolute simultaneity, whose existence he had taken as a self-evident Aristotelian principle, and had also raised objections against abandoning strict causality in quantum theory. This by itself, declares Beth, is already reason enough to reject Hoenen's conception of the relation between science and philosophy.

Beth then finally reveals his own outlook: the task of philosophy of nature is to give a syntactic and semantic analysis of scientific theories. In other words, philosophy of nature and science are not to be thought of as existing side by side on the same level of analysis and therefore as potential rivals; rather, philosophy of nature should be located on a meta-level and should take scientific theories as its given objects of inquiry. Philosophy of nature should provide the analytical and logical tools with the help of which any scientific theory, be it modern or ancient, can be dissected. Just 
as the scientist makes a systematic analysis of the natural world, the philosopher of nature should analyze the finished products of science, theories. In this, he should first of all concentrate on the logical structure of the theory under discussion.

Beth concludes his explanation with a telling passage (Beth 1948a, p. 61). He writes: "Construing the philosophy of nature as indicated above would not only make it possible to treat this discipline in an exact way, it would also make philosophy of nature independent of the influence of world views and ideologies, an influence that today can still be noticed very clearly. This will not be regarded as progress by those who regard world views and ideologies as the very core of philosophy. But those who, like me, wish to consider philosophy of nature as a science, and are, like me, convinced that in science no influence of a world view or an ideology can be tolerated, will think differently."

There thus appears an additional reason for the prominent presence of father Hoenen in Natuurphilosophie: not only was Hoenen a representative of an old-fashioned, aprioristic and basically science-unfriendly philosophy of science, he also served as a symbol of the intrusion of ideology (in this case, catholicism) into the scientific domain. Beth insists that such ideological preconceptions can only harm science and will moreover make it impossible to analyze science in an objective way: they should be exorcized.

The empirical science that is actually analyzed by Beth in Natuurphilosophie and related publications is physics. Beth's detailed philosophy of nature is a philosophy of physics, characterized by the aim of giving an objective and precise logical analysis of physical theories, untarnished by ideological arguments and speculation.

\section{The logical structure of quantum mechanics}

In Chapter II of Natuurphilosophie Beth summarizes the task of his philosophy of physics in more detail: "In the following I shall attempt to show that a physical theory is an interpretable algorithm. For this purpose I shall attempt to formulate, for various physical theories, the corresponding rules $V, D$ and $S$ " (Beth 1948a, p. 53). These rules specify the syntax (the rules $V$ list the form of the allowed expressions, $D$ are the deduction rules) and the semantics, respectively (the rules $S$ are meant as semantic rules à la Tarski). Beth adds: "One could think that an investigation aimed at finding the basic syntactic and semantic rules of physical theories will not be able to penetrate into the deeper problems of the philosophy of nature. This view, I trust, will appear to be incorrect in the following" (Beth 1948a, p. 54).

In Chapter V of Natuurphilosophie the concrete work is begun: first, as a preparation, a logical analysis is made of a fragment of classical mechanics and then attention is directed to the real subject, quantum mechanics. Beth considers the classical mechanics of an harmonic oscillator, i.e. a material point particle moving along a straight line, under the influence of a position-dependent force directed to the origin: $F=-\frac{1}{n} x$ (with $n$ a constant). First rules $V$ are proposed: elementary statements are to have the form $[x, p]_{t}$ (with the intended meaning that the particle possesses position $x$ and momentum $p$ at time $t$ ) and can be concatenated by means of the connectives of the propositional calculus. The usual deduction rules of the propositional calculus are also 
taken over; but they are completed by a new rule, namely " $\left[x+\frac{1}{m} p d t, p-\frac{1}{n} x d t\right]_{t+d t}$ is an immediate consequence of $[x, p]$ ". This specifically mechanical deduction rule expresses the law of motion of classical mechanics, $F=m d^{2} x / d t^{2}$; it expresses the time evolution of $x$ and $p$ during an infinitesimal interval $d t$. As Beth notes, the rule is different from the others because it needs mathematical tools for its expression. In fact, the differential calculus must be presupposed in order to make its formulation possible. One might therefore be inclined to ask whether it is not possible to formulate the new rule in terms of elementary logic. Beth replies that this is unnecessary, even if it were possible, since there is no essential difference of any kind between logical and mathematical tools (Beth 1948a, p. 96).

Finally, the algorithm should be interpreted, and this is done in terms of observation reports. The empirical content of $[x, p]_{t}$ is that at time $t$ the mass point is found to be at position $x$ with velocity $p / m$ (i.e., momentum $p$ ). With the help of the logical connectives more complicated empirical statements can be built up.

At this point Beth raises the question of how the physical quantities $x, t$ and $p$ are actually to be determined; he points out that this must be done with respect to an inertial system (a frame of reference with respect to which the dynamical laws in their standard form can be applied). This remark gives him the opportunity to briefly digress about the status of inertial systems; a problem about which he says that only Einstein, with his general theory of relativity, has been able to solve it (as Beth promises us to explain further in Chapter VII). I believe that this brief aside (and its extensive elaboration in Chapter VII) sheds an interesting light on Beth's attitude. Remember the emphasis Beth had placed on the task of the new philosophy of nature: this should be a sober logical analysis of already given physical theories, not an attempt at founding, changing or criticizing them. Now, classical mechanics, as an historically given theory, does not have a problem of measurement at all: there has never been serious controversy about how to measure $x, t$ and $p$ or about how to identify inertial systems in practice. It is true that there is a history of philosophical debate surrounding the status of inertial systems (starting with the famous Leibniz Clarke/Newton exchange), and it is also true that relativity theory has been relevant in this context; but this is first of all a philosophical debate 'in the old style' concerning the 'essence' of the concepts of space and time, quite independently of the logical analysis of how these concepts actually function within Newtonian theory. There is therefore every appearance that Beth is here looking for a way to find a niche for at least certain more traditional questions within his own rather stringent logical approach. But more about this later.

Beth's analysis of quantum mechanics follows the same general strategy as in the simpler classical mechanics case. The essential difference between the two cases is that quantum mechanics does not use a phase space (like the $(x, p)$-space in the harmonic oscillator example) to represent the states of physical systems, but employs a more complicated mathematical structure, namely a Hilbert space. The states of quantum systems are represented by vectors in this Hilbert space, and physical quantities correspond to operators (in fact, hermitian operators) defined on the same space. In the classical case the value of a physical quantity was given by a function on the phase space (for example, the energy of the harmonic oscillator in the state $(x, p)$ is given by $E=1 / 2 n x^{2}+1 / 2 m p^{2}$ ), but now a physical quantity represented by an operator $\mathbf{M}$ will only have a well-defined value in vector state $\mathbf{x}$ if $\mathbf{x}$ is an eigenvector of $\mathbf{M}$ : 
$\mathbf{M} . \mathbf{x}=m \mathbf{x}$, in which $\mathrm{m}$ is a number representing the value the quantity possesses in this state. This difference in mathematical structure leads to a difference in the logical structure of the theory as Beth defines it.

The elementary statements (we are formulating the rules $V$ now) have the form $\mathbf{p}(p, t)$, with the intended interpretation that the physical quantity $\mathbf{p}$ possesses, at time $t$, the value $p$ (so that $p$ must be an eigenvalue of the operator $\mathbf{P}$ corresponding with $p$ ). Elementary statements corresponding to commuting operators are compatible; otherwise they are complementary. This distinction already introduces an important structural difference in the syntax compared to the classical case.

The deduction rules are the usual rules of the propositional calculus, but now with the restriction that they should only be applied to compatible propositions. In addition to these rules new rules are needed that formalize the mathematical relations in Hilbert space, plus the time evolution of the vector states. For example: "If the operator $\mathbf{P}$ possesses a non-degenerate eigenvalue $p$ with eigenvector $\mathbf{x}$, whereas $\mathbf{x}$ is also eigenvector of $Q$ with eigenvalue $q$, then $\mathbf{q}(q, t)$ is an immediate consequence of $\mathbf{p}(p, t)$." Beth adds this rule plus two other typically quantum mechanical deduction rules to the rules taken from the propositional calculus. The concept of derivability is then defined in the usual way, although with the restriction that the premisses in a derivation should always be compatible.

In a note added before the final publication of the manuscript Beth wrote: "Rereading what I wrote a couple of years ago, I am inclined to think that it is possible to construct the logic of quantum mechanics semantically with the help of the Hilbert space. The deduction rules 7-9 (the added quantum mechanical deduction rulesDD) are not analogous to the rules 1-6 (the deduction rules of the propositional calculus-DD), but rather analogous to the definition of 'entailment'. The interpretation developed in Chapter VII (in terms of observation statements-DD) is therefore not an analogue of semantics" (Beth 1948a, p. 133).

This remark, whose importance was emphasized by Van Fraassen (1970), marks the beginning of what is now known as the semantic approach to the analysis of scientific theories. What Beth is suggesting here is that instead of focussing on syntax and formal deduction, in the style of e.g. Carnap, it is also possible to begin with the mathematical structure of the state space provided by the theory (in the case of classical mechanics this is the phase space, in the case of quantum mechanics the Hilbert space). Elementary statements of the kind we have encountered above can be thought of as corresponding to regions in such a state space, namely the regions containing exactly the states that make the statements in question true. The mathematical structure of the state space determines the relations between such regions, and these induce relations between the corresponding statements; in particular, a relation of entailment can thus be defined. This approach yields a precise Tarskian formal semantics. The relation with observation and measurement results remains important for the empirical interpretation of the theory, of course; but now this becomes the subject of a separate measurement theory that is to link the mathematical entities of the state space to measurement procedures, approximation techniques, etc. The structure of the theory itself, and its logic, is fixed by the mathematical properties of the phase space of the theory.

It is clear from the outset that this semantic approach is much closer to actual scientific practice than the syntactical approach à la Carnap, in which in principle even 
ordinary mathematical-physics reasoning should be reconstructed in terms of logical deductions. The semantic approach promises to make it possible to obtain results of formal analyses that are recognizable to scientists themselves, and the hope seems therefore justified that such philosophical investigations might contribute to answering questions raised within the sciences.

Beth wrote three papers on this new approach (Beth 1948b, 1949, 1960), to which we now turn.

\section{Beth's semantic analysis of quantum mechanics}

In the original manuscript of Natuurphilosophie the deductive logical structure of physical theories was already constructed in such a way that it reflected mathematical reasoning about the relevant state space and the temporal evolution defined in it. The novel element in the new approach is that this syntactical structure is provided with a precise and detailed underpinning in formal semantics, in the form pioneered by Tarski (1935). The difference may seem small, but Beth must certainly have felt that he had made a significant step here. He quickly published two papers containing his new point of view, a very short communication in 1948 (Beth 1948b) and a more substantive one in 1949 (Beth 1949).

The 1948 Synthese paper opens with: "The present study has as its aim to show the applicability of the semantic method, introduced by Alfred Tarski, to the logical analysis of physical theories as started by G. Birkhoff and J. von Neumann, J.-L. Destouches, Paulette Destouches-Février, Hans Reichenbach and Martin Strauss"; which is followed by an extremely succinct (hardly one page) definition of semantic entailment in classical mechanics and quantum mechanics, respectively-without any further comment. The briefness of the paper is remarkable, even if one takes into account that a footnote says that it summarizes a lecture given by Beth; also the neutrality of tone is conspicuous. Perhaps Beth had found out only recently that very similar ideas had been developed by some of the authors he quotes and he did not want to claim too much originality? Indeed, the resemblance between what was proposed, e.g., in a 1938 paper by Strauss (1938) and Beth's new approach is striking.

The 1949 Methodos paper is much more informative. Interestingly, Beth starts by announcing that this paper is meant as a condensed summary of Natuurphilosophie. He adds that the writing of Natuurphilosophie took place in 1943 and 1944 'under the increasing hardships of German occupation', which 'may account for several deficiencies and errors' - the present occasion offers the opportunity to correct a number of these and to insert some new results.

Beth then explains that a major theme of Natuurphilosophie is the increasing discrepancy between science and philosophy, as exemplified by the work of well-known philosophers like H. Dingler, P. Hoenen and J. Maritain. ${ }^{3}$ This discrepancy is one of the main causes of the downfall of contemporary philosophy, Beth continues, which

\footnotetext{
3 Hugo Dingler (1881-1954) was an operationalist philosopher of science, notorious for his fierce opposition to relativity theory and his connections with the so-called Arische Physik; Jacques Maritain (1882-1973) was a noted catholic philosopher and neothomist; Petrus Hoenen we have met before.
} 
seriously menaces the future development of Western civilization. The negative influence that can be discerned in the background is Aristotle's philosophy of science, which asserts that science has to start from self-evident first principles (Beth here credits the identification of this 'evidence postulate' to Scholz (1930)) - according to Beth, this Aristotelian postulate permeates all traditional philosophy. As a remedy Beth recommends the development of an up-to-date philosophy of natural science that should analyze the form and semantics of scientific theories, instead of developing speculative alternatives or venturing solutions to scientific problems. Beth concludes this introductory part by pointing out that the semantic method, which he here credits to Tarski (1935), Von Neumann (1932), Strauss (1938) and Destouches (1942), can be a very great help in such logical analyses of physical theories.

There follow the usual definitions of semantic entailment for classical mechanics and quantum mechanics. For the case of quantum mechanics, the basic idea is that "a phrase (a) is a consequence of the phrases (b), if, and only if, any vector, which satisfies the phrases (b), satisfies also the phrase (a)" (Beth 1949, p. 182). Here, like in Natuurphilosophie, a vector $\mathbf{x}$ in the Hilbert space is taken to satisfy a phrase $\mathbf{m}[m, t]$ (quantity $\mathbf{m}$ has the value $m$ at time $t$ ) if and only if $\mathbf{M} \cdot \mathbf{x}=m \mathbf{x}$, where $\mathbf{M}$ is the hermitian operator corresponding to $\mathbf{m}$.

Something new follows at this point: 'a few words' about the 'well-known EinsteinPodolsky-Rosen paradox'. Beth does not explain the paradox, which I shall therefore briefly describe. Einstein and coworkers had proposed a thought experiment, in 1935, with the purpose of showing that quantum mechanics is unable to give a complete description of reality; quantum mechanics should according to these authors therefore not be considered the last word in physical theory. The experiment is about a twoparticle system that possesses a well-defined total momentum $(p)$ and also a welldefined difference of positions of the components $(q)$; this is possible according to quantum mechanics because there exist quantum states that are joint eigenstates of $\mathbf{P}_{1}+\mathbf{P}_{2}$ and $\mathbf{Q}_{1}-\mathbf{Q}_{2}$, with eigenvalues $p$ and $q$, respectively. Now, let the particles travel very far apart and then perform a position measurement on particle 1; let the result be $q^{\prime}$. We can then predict, with certainty, what a subsequent measurement on particle 2 , if performed, would yield, namely $q^{\prime}-q$. Alternatively, we could have performed a momentum measurement on particle 1; let us assume that in that case we would have found a value $p^{\prime}$. That result would have made it possible to predict, again with certainty, the momentum value of particle 2 to be found in a subsequent measurement: $p-p^{\prime}$. Now Einstein reasons as follows. For the far-away particle 2 it does not make any difference whatsoever whether or not any measurement is performed on particle 1: indeed, there can be no causal contact between the two wings of the experiment because there is not enough time for a signal to cover the enormous mutual distance. Therefore, the values of position and momentum of particle 2 about which we could obtain certainty if we were to do certain experiments on particle 1, must be present in particle 2 even if we refrain from doing any experiment at all. That means that particle 2 must possess both a well-defined value for its momentum and for its position, quite independently of what is going on at the position of particle 1. However, there do not exist quantum states that are joint eigenstates of both the position and momentum operator. Therefore, quantum mechanics is incomplete: these states must apparently exist in reality but quantum mechanics does not contain them. 
Beth deals with the thought experiment very briefly, as follows. He says: Einstein, Podolsky and Rosen derive the phrases (a):

$$
\begin{array}{r}
\mathbf{p}_{2}\left[p-p^{\prime}, t^{\prime \prime}\right] \\
\mathbf{q}_{1}\left[q+q^{\prime}, t^{\prime \prime}\right]
\end{array}
$$

from the phrases (b):

$$
\begin{array}{r}
\left(\mathbf{p}_{1}+\mathbf{p}_{2}\right)\left[p, t^{\prime}\right] \\
\left(\mathbf{q}_{1}-\mathbf{q}_{2}\right)\left[q, t^{\prime}\right] \\
\mathbf{p}_{1}\left[p^{\prime}, t^{\prime \prime}\right] \\
\mathbf{q}_{1}\left[q^{\prime}, t^{\prime \prime}\right]
\end{array}
$$

But this derivation, Beth continues, is trivial: there is no state satisfying all the phrases (b). In testing a physical theory such a trivial consequence can be of no use. "These short comments may be sufficient to show the utility of a logical analysis of quantum mechanics."

Unfortunately, the formulas in Beth's paper (as reproduced above) are incorrect. They are inconsistent and obviously involve a printing error-but since no clarification is provided about the exact set-up Beth has in mind it is not completely trivial how to correct them. Given his later publication (Beth 1960) it is almost certain, however, that the last line of (b) is meant to read: $\mathbf{q}_{2}\left[q^{\prime}, t^{\prime \prime}\right]$. This amendment makes the algebra consistent and is in accordance with the explicit statements about the EPR experiment made by Beth (1960). It also means, however, that Beth is here misrepresenting Einstein's argument, which as explained above is not about a $\mathbf{p}$ measurement performed on particle 1 and a simultaneous $\mathbf{q}$ measurement on particle 2. This deviation from Einstein's strategy spoils the very idea of the thought experiment.

But even if we attempt to correct for this, and try to formalize Einstein's own argument in Beth's language, the result remains disappointing. The reason is that the only thing that is and can be achieved by Beth's formalization is a redescription of what quantum mechanics already tells us, but now in his specified formal language. The essential observation made by Beth, namely that there are no quantum mechanical states with both a precise momentum and a precise position and that we cannot base any valid quantum mechanical derivation on the supposed existence of such states, is what we knew all along; this was exactly Einstein's reason for concluding that quantum mechanics is incomplete! What Beth demonstrates is that the quantum mechanical treatment is internally consistent-but this was not contested by Einstein and was not the target of the EPR thought experiment. Einstein wanted to criticize quantum mechanics on the basis of criteria brought in from outside of quantum mechanics, and this can evidently not be captured by a rendering in formal language of what quantum mechanics itself says about the issue.

The 1960 Synthese article (Beth 1960) does not change the situation. It is an almost verbatim reproduction of the part of the 1949 paper that deals with semantic entailment and EPR, but now with some words of explanation added about the measurements 
performed in the experiment; from this it becomes clear that Beth indeed thought that the experiment referred to simultaneous measurements on both wings.

This by itself is not meant as a severe criticism: there has been endless confusion in the literature about EPR and Beth's variation on the original set-up is discussed by many others as well. What is more serious is that in principle Beth's formal approach will never yield more than a correct quantum mechanical treatment of the EPR experiment — and this, by definition, will not do justice to Einstein's argument. We are here facing a possible general weakness of Beth's approach: if the idea were to be taken literally that the philosophy of physics should refrain from all 'speculation' and should content itself with merely describing the mathematical structure of existing theories, a not very exciting activity would remain that would exclude important parts of the philosophy of physics as it is and has been successfully practiced. Think of Huygens, Leibniz or Mach criticizing Newton; or, indeed, Einstein, de Broglie or Bohm criticizing quantum theory; in these cases what is at stake is not a lack of clarity with regard to the logical structure of the criticized theory, but rather a clash between the theory and certain 'metaphysical' desiderata. However, there are reasons to suppose that Beth himself did not really intend to be this strict in general, but rather was willing and perhaps even anxious to create room for at least some of these 'metaphysical' debates. His interest in the discussion surrounding the nature of space and the status of inertial systems (already mentioned in Sect. 3) constitutes an example.

\section{Beth on absolute space}

Under the pretext of inquiring into the relation between theory and observation, in Chapter VII of Natuurphilosophie Beth intervenes in the Newton-Leibniz-MachEinstein discussion about absolutist versus relationist conceptions of space. He starts by reminding us that the empirical content of a physical theory derives from the observation of events, that events are ordered in the space-time continuum, and continues by noting that the relations between such space-time descriptions as given by different observers are not at all trivial. This then is taken as a useful leg up to a review of Newton's ideas, Mach's criticism of them and finally relativity theory. In the whole discussion Beth's Machian empiricist sympathies clearly shine through. The idea of an independent space or spacetime is depicted as a relic of Aristotelian science, and relativity theory is characterized as the pinnacle of the history of physics that has put the peripatetic viewpoint definitively behind us. In an interesting 12-pages appendix to his chapter Beth develops some ideas of his own on the technicalities of the issue: "In the following I wish to show how one could develop Mach's ideas mathematically."

The idea is to start from Newton's theory, namely the Newtonian equation of motion for a point mass in a frame that is at rest in absolute space. Beth subsequently writes this equation down in the coordinates of a uniformly rotating frame, which leads to a more general form, involving a centrifugal and a Coriolis force. He then rewrites this equation as follows (in slightly modified notation):

$$
\vec{a}=\nabla \varphi+\vec{v} \times(\nabla \times \vec{A})+\frac{\partial \vec{A}}{\partial t}
$$


in which $\vec{a}$ is the acceleration of the point mass, $\vec{v}$ its velocity, $\varphi$ the gravitational potential (Beth only considers gravitational forces) and $\vec{A}$ a 'vector potential' representing the Coriolis and centrifugal forces. Beth comments: "We have now brought the basic equations of mechanics and gravitation into a form that does not only hold in a frame resting in absolute space, but also in our new frame. Consequently, we from now on decline to accept the privileged status of absolute space and consider all our frames as equivalent; I will call such a frame a Mach frame" (Beth 1948a, p. 171).

If this argument is meant as an argument for the physical equivalence of the considered frames, it is fallacious. The question is not whether it is possible to write the equations of motion in such a form that they look the same in every frame, like in Eq. 1. If that were the task, the solution would be simple enough: the derivation of Eq. 1 is not even needed, for we could just take the general equation of motion involving Coriolis and centrifugal forces - this equation already has a form that is the same in each and every frame. But this manoeuvre obviously does not make all frames physically equivalent: the value of the centrifugal and coriolis forces is still different in different frames, and inertial frames keep distinguishing themselves because in them these forces vanish. Taking a step back, we can easily convince ourselves that a mere rewriting of equations, so that their formal appearance changes, will do nothing to change physical differences between frames of reference.

It must immediately be added that the confusion between form invariance of equations and physical equivalence of reference systems is an old one; it began with Einstein's use of the 'principle of general covariance' and led to extensive discussions already in the first years of general relativity (see for more on this history, e.g., Dieks (2006)). Beth apparently was not aware of the corresponding physics literature. But, very much to Beth's credit and testifying to his sharp insight, on pages 172-174 he develops some of the relevant doubts himself. In response to his own qualms in accepting the above argument as an argument for physical equivalence, he first tries to salvage Machian relationism by proposing a speculative theory in which $\vec{A}$ from Eq. 1 is not just an expression of a priori given inertial forces, but becomes a dynamic quantity; he writes down a possible differential equation for $\vec{A}$ that connects it to the distribution of matter in the universe. But Beth quickly (and cleverly) follows this up with the remark that even in such a theory boundary conditions for the equation determining $\vec{A}$ will have to be specified, which revives the threat of an a priori privileged position of some frames. At this point Beth abandons his own project and turns to Einstein's general theory of relativity.

After an explanation of the main principles of Einstein's general theory, Beth comes to the correct conclusion that even this theory does not abolish spacetime as something that exists independently of matter. Even here there is a remnant of Newtonian absolute space: although the geometrical properties of general relativistic spacetime are influenced by matter, there exist non-trivial models of the theory (solutions of the Einstein field equations) in which there is no matter at all. These are 'empty spacetimes', whose existence as models of general relativity demonstrates that general relativistic spacetime has a life of its own, independently of matter.

In a short concluding paragraph Beth finally informs us that the whole question has now been laid to rest: Einstein's variation from 1917 on his own theory, which introduces the so-called cosmological constant, is in full agreement with Mach's ideas 
and it is this theory that is used in cosmological research, like that of de Sitter. Unfortunately, this paragraph is based on a misunderstanding. It is true that Einstein introduced his 1917 equations (the ones with the cosmological constant) in the hope and expectation that the resulting theory would be Machian, but de Sitter quickly showed, still in 1917, that even Einstein's new theory had non-trivial spacetime solutions in which there is no matter-so that spacetime in Einstein's new theory can still be empty and is therefore not reducible to matter.

\section{Beth as a philosopher of physics}

With hindsight, we can observe that scientific philosophy was internationally 'in the air' in the decade preceding the second world war. Both new results in logic (Tarski) and the anti-metaphysical attitude promulgated by the members of the Vienna Circle had been influential; and people like Carnap, Reichenbach, Strauss, Birkhoff and von Neumann had already applied some of the corresponding insights to the philosophical analysis of physics. New journals had been founded that reflected the new zest, like Erkenntnis and Synthese. These modern philosophical endeavors were well received among Dutch physicists, at least the philosophically interested ones of them. However, the philosophy of science that was officially represented at the Dutch universities had not followed the new trend, at least not as far as the philosophy of the empirical sciences was concerned. On the contrary, neothomism, which had been a significant influence also in other European countries, had acquired a particularly high profile in the Netherlands as a consequence of the specifically Dutch catholic emancipation campaign.

Again with hindsight, we may ascertain that neothomism was already past its heyday at the end of the war years. In Italy for example, the country from which Petrus Hoenen wrote, considerable resistance had arisen against the "philosophy of the great systems' and two new journals had been founded to promote a new, sciencebased alternative (Analisi, 1945 and Sigma, 1947) (Somenzi 1969). In 1949 these two journals merged to form the journal Methodos, to whose opening issue Beth contributed his paper 'Towards an up-to-date philosophy of the natural sciences' (which was, as we have seen, a summary of his Natuurphilosophie). At this time, Beth evidently had already been recognized as a leading exponent of the international scientific philosophy movement; a stature he had quickly achieved after his appointment in Amsterdam. In the Netherlands his star had risen even higher: he had become the representative of the new logic and science oriented direction in philosophy.

Beth's success was without doubt at least partly due to his fierce (although, as we have seen, sometimes a bit rhetorical) opposition to traditional philosophy. His unremitting fight for modernity (e.g., Beth 1943, 1946a,b, 1947) was especially welcome in the Dutch situation, in which several special chairs for neothomist philosophy had recently been established. Beth's activities found a favorable response and marked the beginnings of a solid Dutch academic tradition in scientific philosophy.

As far as the more technical contents of Natuurphilosophie are concerned, the part that has probably gathered most following is the application of formal semantics to physical theories. Although only worked out in Beth's later publications, the gist 
of the method is clearly already present in the 1948 book. In particular through its extension by Van Fraassen (1970), Beth's approach has received wide international attention and has now become the standard in formal analyses of physics. Although similar semantic approaches were also pioneered by others, I think it is safe to say that Beth developed his ideas independently and can be credited with a major part of its present-day success.

In comparison, Beth's contributions to specific problems in the foundations and philosophy of physics have been less successful- he remained a bit of an outsider here and was first of all a logician and philosopher of mathematics. But his Natuurphilosophie and later publications clearly testify to his great enthusiasm for the developments in modern physics, and to his conviction that their analysis possesses a deep philosophical relevance.

Open Access This article is distributed under the terms of the Creative Commons Attribution Noncommercial License which permits any noncommercial use, distribution, and reproduction in any medium, provided the original author(s) and source are credited.

\section{References}

Beth, H. J. E. (1932). Newton's “Principia”. Groningen: Noordhoff.

Beth, E. W. (1935). Rede en aanschouwing in de wiskunde. Dissertation Utrecht University, Noordhoff, Groningen.

Beth, E. W. (1938). Het wetenschapsbegrip bij Nietzsche en bij de neo-positivisten. De Gids, 102, $273-286$.

Beth, E. W. (1943). Verleden en toekomst der wetenschappelijke wijsbegeerte. De Gids, 107, 46-67.

Beth, E. W. (1946a). De strekking en het bestaansrecht der metaphysica in verband met de toekomst der wijsbegeerte. Inaugural lecture Amsterdam, Noordhoff, Groningen

Beth, E. W. (1946b). Metaphysica en wetenschap. De Gids, 109, 83-91.

Beth, E. W. (1946c). Historical studies in traditional philosophy. Synthese, 5, 248-260.

Beth, E. W. (1947). Exact-wetenschappelijke wijsbegeerte in Nederland. Nieuw Tijdschrift voor Wiskunde, 35, 100-104.

Beth, E. W. (1948a). Natuurphilosophie. Gorinchem: Noorduijn.

Beth, E. W. (1948b). Analyse sémantique des théories physiques. Synthese, 7, 206-207.

Beth, E. W. (1949). Towards an up-to-date philosophy of the natural sciences. Methodos, 1, 178-185.

Beth, E. W. (1950). Wijsgerige Ruimteleer. Antwerpen: Standaard-Boekhandel.

Beth, E. W. (1960). Semantics of physical theories. Synthese, 12, 172-175.

Destouches, J. L. (1942). Principes fondamentaux de physique théorique. Paris: Editions Hermann \& Cie.

Dieks, D. (2006). Another look at general covariance and the equivalence of reference frames. Studies in History and Philosophy of Modern Physics, 37, 174-191.

Hoenen S.J., P. (1928). De groote crisis der physische wetenschappen. Studiën, 60, 173-190.

Hoenen S.J., P. (1930). Cosmologia. Rome: Gregoriana.

Hoenen S.J., P. (1940). Philosophie der Anorganische Natuur. Antwerpen: Standaard-Boekhandel.

Scholz, H. (1930). Die Axiomatik der Alten. Blätter für Deutsche Philosophie, 4, 259-278.

Somenzi, V. (1969). La filosofia e la metodologia della scienza oggi in Italia. Man and World, 2, $285-295$.

Strauss, M. (1938). Mathematics as logical syntax — a method to formalize the language of a physical theory. Erkenntnis, 7, 147-153.

Tarski, A. (1935). Der Wahrheitsbegriff in den formalisierten Sprachen. Studia Philosophica, 1, 261-405.

Van Berkel, K. (1996). Dijksterhuis. Amsterdam: Bert Bakker.

Van Fraassen, B. C. (1970). On the extension of Beth's semantics of physical theories. Philosophy of Science, 37, 325-339.

Von Neumann, J. (1932). Mathematische Grundlagen der Quantenmechanik. Berlin: Springer. 